\title{
Study of Vibration Milling for Improving Surface Finish of Difficult-to-Cut Materials
}

\author{
Vytautas Ostasevicius - Rimvydas Gaidys - Rolanas Dauksevicius* - Sandra Mikuckyte \\ ${ }^{1}$ Kaunas University of Technology, Faculty of Mechanical Engineering and Mechatronics, \\ Institute for Hi-Tech Development, Lithuania
}

\begin{abstract}
This work studies the influence of high-frequency excitation of a cutting tool during end milling of workpieces made of difficult-to-cut metallic alloys. It is demonstrated that high-frequency vibrations superimposed onto the continuous movement of the tool lead to milling process stabilization with superior surface finish in comparison to conventional machining. A finite element model of the vibration milling tool was built and verified experimentally. The model treats the tool as an elastic pre-twisted structure (mill cutter) characterised by its natural vibration modes. The resonance frequencies of the axial vibration mode of cutters of two different lengths were predicted numerically and subsequently used for excitation of the vibration milling tool during cutting experiments. Qualitative and quantitative characterization of the surface quality of the machined stainless steel and titanium alloys was performed. Measurement results have confirmed that excitation of a specific tool mode is a prerequisite for achieving maximal efficiency of the vibration milling process. Statistical analysis of the collected roughness measurement data identified factors that most significantly contribute to the improved surface finish of the workpieces.
\end{abstract}

Keywords: vibration cutting, finite element model, pre-twisted cantilever, axial mode, roughness

\section{O INTRODUCTION}

Controlling vibration phenomena in production machines is one of the approaches for improving their efficiency. This also applies to cutting tool vibrations generated during machining, when the magnitude of the vibrations directly influences workpiece surface quality. Continuous efforts to enhance cutting performance have revealed that machining quality may be improved if a tool is assisted with high-frequency vibrations. During the resulting vibration cutting process [1], the tool periodically loses contact with the chip leading to a reduction in machining forces, friction, and temperature in the cutting zone and the formation of thinner chips, as well as simultaneously preventing generation of micro-cracks on the cutting edge and workpiece surface. As a consequence, this improves cutting stability, surface finish, and tool life when compared to conventional machining [2].

The authors of this paper previously reported on an approach for the reduction of workpiece surface roughness by exciting higher-order transverse modes in the vibration turning tool [3]. Twardowski et al. [4] analysed various factors affecting surface roughness after end milling of hardened steel in high-speed milling. The work also involved analysis of surface profile charts in terms of vibrations and cutting force components. Chen et al. [5] studied the effect of surface roughness of end mills on cutting performance in the case of high-speed machining. A novel optimization design approach to the processing parameters for high-speed cutting has been proposed. Taylan et al. [6] considered the wear performance of $\mathrm{CBN}$ and
TiN-coated CBN cutting tools during face milling of $61 \mathrm{HRC}$ hardened $90 \mathrm{MnCrV} 8$ tool steel workpieces. Kubiak et al. [7] evaluated the effect of topographical parameters on wettability and spreading phenomenon by using statistical covariance analysis. Anisotropic surfaces were prepared by abrasive polishing on aluminum, titanium, copper alloys, and steel. Imani and Moosavi [8] have proposed a new force model of torsional-axial and transverse vibration for drilling, which was validated experimentally. Simulation of torsional-axial vibrations is accomplished using Bayly's model, which is based on the fact that a twist drill lengthens when it »untwists《.

A number of studies have been conducted to monitor vibrations during the milling operation. The results of Zhang and Chen [9] indicate the feasibility of using cutting vibration amplitudes and frequency peaks in two directions, $\mathrm{X}$ and $\mathrm{Y}$, for the monitoring of tool condition during end milling operations. Seguy et al. [10] examined the relationship between chatter instability and surface roughness evolution for thin wall milling. An explicit numerical model was developed for analysis of modal interactions. It takes into account the coupling mode, the modal shape, and the fact that a tool may have a ploughing effect.

Grazeviciute et al. [11] presented measurement results for surface roughness and chatter frequency in the case of the vibration milling of aluminum AlMgSi0.5 (6060) and plastic PA6. The results indicate that the surface of he aluminum alloy after vibration-assisted milling is smoother than after conventional milling. However, no difference was detected in the case of milling plastic material. 
Promising results obtained by the authors (us) during research work on vibration turning [3] prompted us to focus on the milling operation. The research methodology employed in this work is based on the combined numerical-experimental approach used in [3], which is extended here to the case of vibration milling. The research objective of this paper is the vibration milling of difficult-to-cut alloys with the aim of determining the influence of tool excitation conditions on the surface finish of workpieces through the application of qualitative and quantitative surface characterization methods.

\section{NUMERICAL ANALYSIS OF THE VIBRATION MILLING TOOL}

\subsection{Structure of the Developed Tool Prototype}

Fig. 1a provides a structural diagram of a prototype of the vibration milling tool developed at the Kaunas University of Technology. A ring-shaped piezoelectric transducer 8 is embedded in the vibration tool assembly consisting of components 2 to 9 . The transducer, powered by collector rings 4 , is used to excite high-frequency vibrations in the cutting edge of the mill cutter 10. A horn 9 with chuck is fitted onto the end of the assembly in order to augment cuttertip vibration amplitude, which may reach up to 20 $\mu \mathrm{m}$ in this case. The vibration milling tool operates in a resonance mode: its length is equal to the integral number of half-wavelengths (Fig. 1b). The tool is mounted in a standard Weldon holder 1 at a nodal point, thereby preventing vibration energy losses by dissipation into the machine tool body.

\subsection{Finite Element Modelling}

\subsubsection{Assumptions}

Laboratory testing of the vibration milling tool has demonstrated that cutter dynamics has a negligible effect on the axial vibrations generated by the tool. This finding justified our model reduction approach, which assumed that the actual excitation provided by the piezoelectric transducer may be represented as an equivalent base excitation, which is imposed on the cutter at the place where it is clamped in the chuck. It should be noted that the vibration tool constitutes a linear dynamic system, therefore its vibrational characteristics may be established by analyzing a numerical model of a single cutter with the boundary conditions that are equivalent to those of the actual vibration tool. Therefore, a structurally complex transducer assembly was discarded in the model resulting in a lower number of degrees of freedom (DOFs), which, in turn, reduced the computational time of simulations.
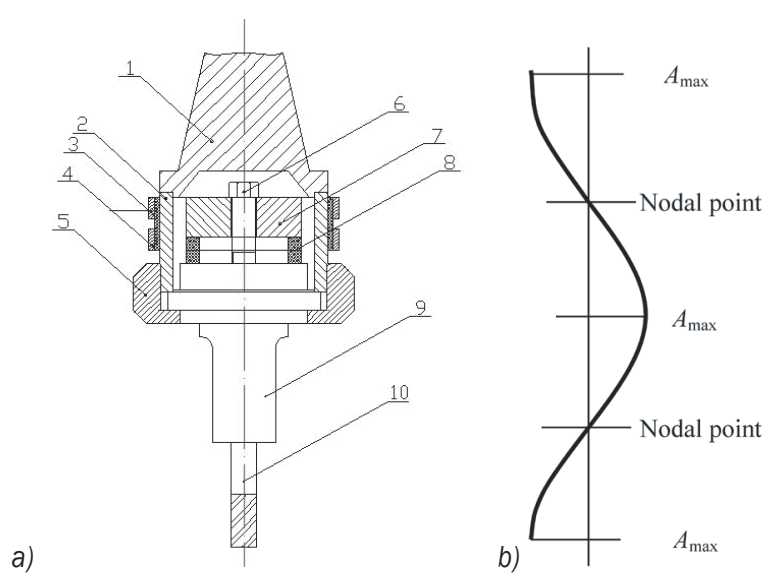

Fig. 1. a) Structural diagram of the vibration milling tool; and b) schematics of its operation principle: 1 - standard holder (Weldon) DIN 6359, 2 - cylinder, 3 - textolite cylinder, 4 - collector rings, 5 - nut, 6 - bolt, 7 - collet, 8 - piezoceramic rings, 9 - horn, 10 - cutter

\subsubsection{Model Formulation}

SolidWorks software was used for preparation of the 3D models of a mill cutter shaped as a pre-twisted cantilever (Fig. 2a). After exporting to FEM software ANSYS, the models were imposed with the relevant boundary conditions (Figs. $2 \mathrm{~b}$ and c). The main properties of FE models: cutter length $l=96 \mathrm{~mm}$ and $l=74 \mathrm{~mm}$, diameter $d=10 \mathrm{~mm}$, density $\rho=8000 \mathrm{~kg} /$ $\mathrm{m}^{3}$, Young's modulus $E=207 \mathrm{GPa}$, Poisson's ratio $n$ $=0.3$. The models were meshed with tetrahedral finite elements SOLID92 characterised by 3 DOFs per node.

A Cartesian coordinate system was adopted for modal analysis, while for harmonic and transient simulations a cylindrical system was chosen with the following DOFs: displacements $\mathrm{z}$ along the cutter rotational axis (axial direction), displacements $r$ orthogonal to the axis (transverse direction), and rotations $\varphi$ about the rotational axis (torsional direction).

It was essential to accurately reproduce the actual conditions of elastic cutter clamping in the chuck since they may have a tangible influence on cutter dynamics due to variable clamping force, temperature, etc. To this end, a sub-model consisting of elastic links was inserted into the cutter model. Spring elements COMBIN14 were selected for modelling the contact interaction in the zones where the cutter is mounted in a chuck (the cutter was secured with three bolts located around the shank at $120^{\circ}$ angles). The cutter 

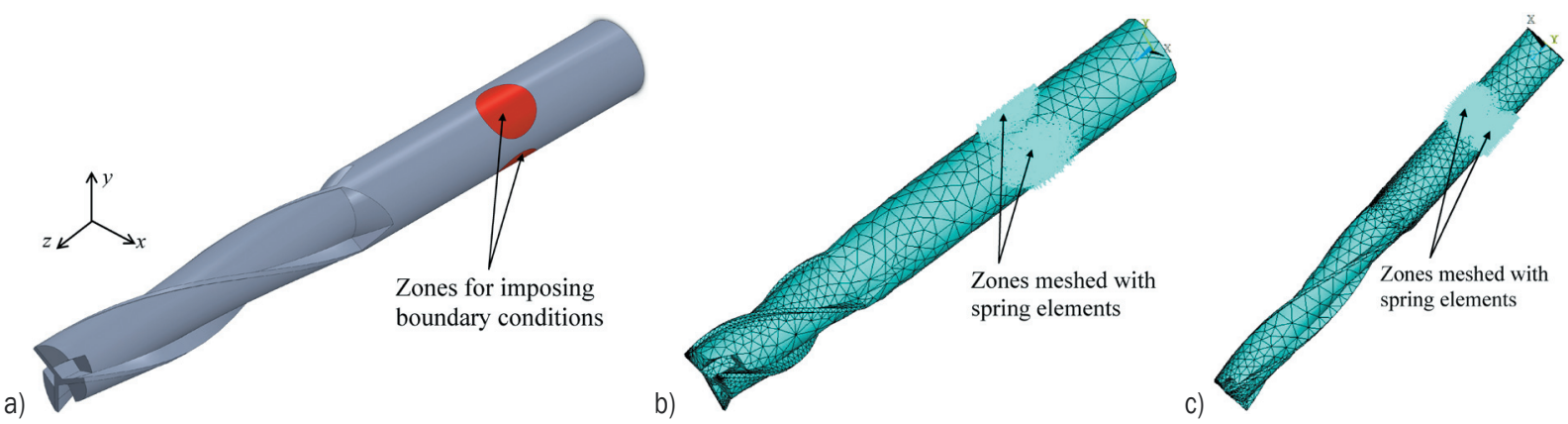

Fig. 2. a) SolidWorks model of a mill cutter with marked zones where boundary conditions are set; ANSYS finite element model of b) $74 \mathrm{~mm}$; and c) $96 \mathrm{~mm}$ length end mill with designated zones that are meshed with spring elements COMBIN14

was fixed elastically with longitudinal spring elements $k_{r}, k_{z}$ and torsional elements $k_{\varphi}\left(k_{r}, k_{z}\right.$ stiffness in transverse and axial directions, respectively, $k_{\varphi}$ - torsional stiffness). These link elements were placed at each node belonging to the three zones located on the shank (Fig. 2a). One node of a spring element was connected to the respective zone node, while the other element node was either constrained (during modal analysis) or was imposed with a base excitation in the axial direction (during harmonic and transient analyses). The developed FE model consists of about 6000 elements SOLID92 and 250 elements COMBIN14 with a total of ca. 30000 DOFs.

The dynamics of the cutter are described by the equation of motion in a block form by considering that the base motion law is known and is defined by the nodal displacement vector $\left\{U_{K}(t)\right\}$ :

$$
\begin{aligned}
& {\left[\begin{array}{ll}
{\left[M_{N N}\right]} & {\left[M_{N K}\right]} \\
{\left[M_{K N}\right]} & {\left[M_{K K}\right]}
\end{array}\right]\left\{\begin{array}{l}
\left\{\ddot{U}_{N}\right\} \\
\left.\ddot{U}_{K}\right\}
\end{array}\right\}+} \\
& +\left[\begin{array}{ll}
{\left[C_{N N}\right]} & {\left[C_{N K}\right]} \\
{\left[C_{K N}\right]} & {\left[C_{K K}\right]}
\end{array}\right]\left\{\begin{array}{l}
\left\{\dot{U}_{N}\right\} \\
\left.\dot{U}_{K}\right\}
\end{array}\right\}+ \\
& +\left[\begin{array}{ll}
{\left[K_{N N}\right]} & {\left[K_{N K}\right]} \\
{\left[K_{K N}\right]} & {\left[K_{K K}\right]}
\end{array}\right]\left\{\begin{array}{l}
\left\{U_{N}\right\} \\
\left\{U_{K}\right\}
\end{array}\right\}=\left\{\begin{array}{l}
\{0\} \\
\{R\}
\end{array}\right\},
\end{aligned}
$$

where $\left\{U_{N}(t)\right\},\left\{U_{K}(t)\right\}$ - nodal displacement vectors representing displacements of free nodes and baseexcited nodes, respectively; $[M],[K],[C]-$ mass, stiffness and damping matrices respectively; $\{R\}-$ vector representing reaction forces of the base-excited nodes.

The displacement vector of the unconstrained nodes is expressed as:

$$
\left\{U_{N}\right\}=\left\{U_{N r e l}\right\}+\left\{U_{N k}\right\},
$$

where $\left\{U_{\text {Nrel }}\right\}$ is a component of the relative displacement with respect to the moving base displacement $\left\{U_{N k}\right\}$.

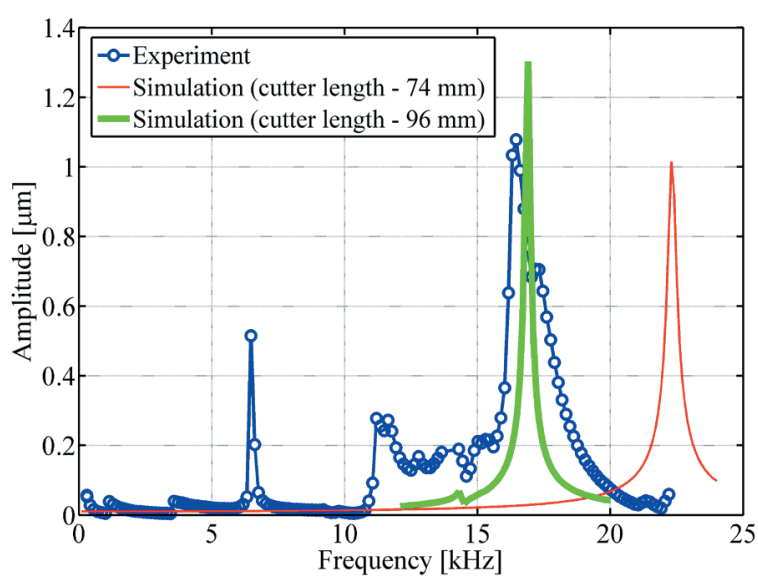

Fig. 3. Experimental frequency response of the cutter in the axial direction and the corresponding numerical responses

Vectors $\left\{U_{K}\right\}$ and $\left\{U_{N k}\right\}$ correspond to rigidbody displacements exerting no internal elastic forces within the structure. A proportional damping approach is adopted in the form $[\mathrm{C}]=\alpha[M]+\beta[K]$, where $\alpha$ and $\beta$ denote Rayleigh damping coefficients. The final equation in a matrix form is as follows:

$$
\begin{aligned}
& {\left[M_{N N}\right]\left\{\ddot{U}_{N \text { rel }}\right\}+\left[C_{N N}\right]\left\{\dot{U}_{N r e l}\right\}+\left[K_{N N}\right]\left\{U_{\text {Nrel }}\right\}=[\widehat{M}],} \\
& \text { where }[\widehat{M}]=\left[M_{N N}\right]\left[K_{N N}\right]^{-1}\left[K_{N K}\right]-\left[M_{N K}\right],
\end{aligned}
$$

where the left-hand side of the equation contains matrices of the structure constrained in the nodes of applied base excitation, while the right-hand side denotes a vector of inertial forces acting on each node as a result of the base excitation. 


\subsubsection{Experimental Verification}

The model was verified experimentally in order to confirm that it is able to accurately predict the dynamic characteristics of the cutter that is excited by the vibration milling tool. The level of coincidence between the measured and simulated frequency responses was used to characterise model accuracy.

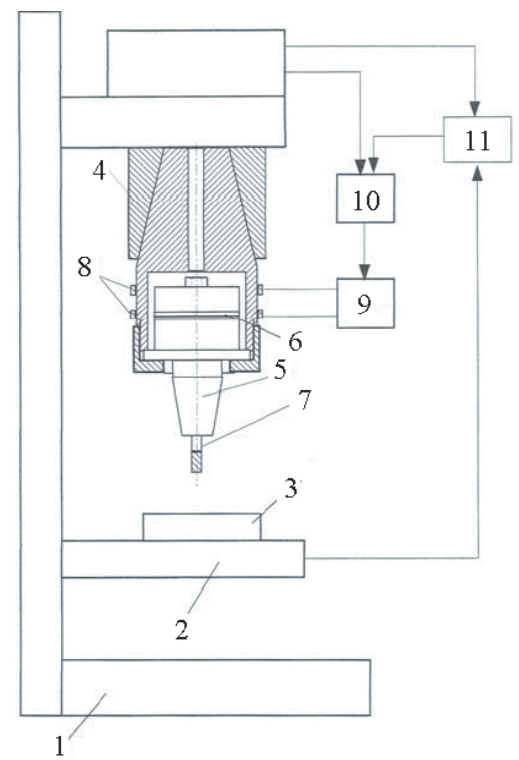

Fig. 4. Scheme of experimental set-up for vibration milling: 1 machine construction, 2 desk, 3 workpiece, 4 vibration tool holder DIN 6359, 5 horn, 6 piezoceramic rings, 7 cutter, 8 collector rings, 9 generator, 10 amplifier, and 11 multimeter

The vibrational response of the cutter is largely predetermined by its boundary conditions. Therefore the model was adjusted by varying the stiffness of the spring elements until an acceptably close agreement between the numerical and experimental resonant frequencies was obtained. Fig. 3 illustrates a comparison between the simulated and measured frequency responses of the cutter in the axial direction (the experimental curve represents the cutter response to a harmonic excitation with a constant relative amplitude).
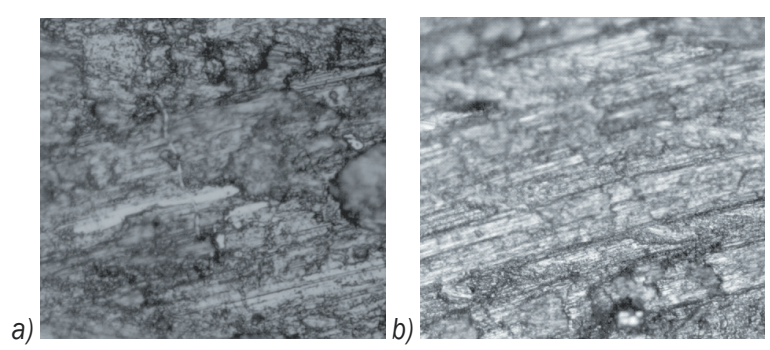

Fig. 5. Photos of surfaces of machined workpieces

(50× magnification): a) after conventional milling and b) after vibration milling

The responses in Fig. 3 reveal that the major measured and simulated resonance peaks coincide fairly well. The accuracy of the FE model is evaluated using the relative error, which is calculated using numerical and experimental values of the resonant frequencies. The relative error was determined to be less than $2 \%$, which confirms the accuracy of the developed FE model.

Resonance frequencies of the axial vibration mode of mill cutters of two different lengths were calculated using the FE model, yielding $14.05 \mathrm{kHz}$ for the $74 \mathrm{~mm}$ cutter and $18.4 \mathrm{kHz}$ for the $96 \mathrm{~mm}$ cutter.

Table 1. Surface finish results of milled workpieces using a $74 \mathrm{~mm}$ length cutter

\begin{tabular}{lccccccccc}
\hline & \multicolumn{7}{c}{ Numbers of measurement $[\mu \mathrm{m}]$} & & \\
\hline Materials and milling type & 1 & 2 & 3 & 4 & 5 & 6 & Average $[\mu \mathrm{m}]$ & Difference [\%] \\
\hline Stainless steel (no vibrations) & 0.61 & 0.56 & 0.75 & 0.87 & 0.53 & 0.58 & 0.65 & \multirow{2}{*}{21.54} \\
\hline Stainless steel (with vibrations) & 0.48 & 0.39 & 0.67 & 0.49 & 0.43 & 0.60 & 0.51 & 0.55 \\
\hline Titanium (no vibrations) & 0.60 & 0.60 & 0.56 & 0.53 & 0.47 & 0.56 & \multirow{2}{*}{6.33} \\
\hline Titanium (with vibrations) & 0.49 & 0.70 & 0.46 & 0.50 & 0.47 & 0.49 & 0.52 & \\
\hline
\end{tabular}

Table 2. Surface finish results of milled workpieces using a $96 \mathrm{~mm}$ length cutter

\begin{tabular}{lccccccccc}
\hline \multicolumn{10}{c}{ Numbers of measurement $[\mu \mathrm{m}]$} \\
\hline Materials and milling type & 1 & 2 & 3 & 4 & 5 & 6 & Average [ $\mu \mathrm{m}]$ & Difference [\%] \\
\hline Stainless steel (no vibrations) & 1.17 & 1.14 & 1.15 & 1.17 & 1.21 & 1.11 & 1.16 & \multirow{2}{*}{22.59} \\
\hline Stainless steel (with vibrations) & 1.00 & 0.86 & 0.91 & 0.78 & 0.95 & 0.88 & 0.90 & \\
\hline Titanium (no vibrations) & 0.76 & 1.30 & 0.88 & 0.86 & 1.01 & 0.85 & 0.94 & \multirow{2}{*}{12.54} \\
\hline Titanium (with vibrations) & 0.83 & 0.85 & 0.85 & 0.80 & 0.80 & 0.82 & 0.83 & \\
\hline
\end{tabular}




\section{CHARACTERISATION OF WORKPIECE SURFACE QUALITY}

\subsection{Evaluation of Surface Roughness}

A series of conventional and vibration-assisted milling experiments were performed in order to determine the effectiveness of the vibration milling tool with respect to improvement in surface quality. Fig. 4 provides a schematic representation of the measurement setup. Experiments were carried in $\mathrm{CNC}$ milling center DMU 35M with workpieces made of stainless steel (1.4301) and titanium (GOST 22178-1976) without the use of cooling-lubricating fluids.

Two end mills of different length $74 \mathrm{~mm}$ (length of working part is $22 \mathrm{~mm}$ ) and $96 \mathrm{~mm}$ (length of working part is $48 \mathrm{~mm})$ but of the same diameter (10 $\mathrm{mm}$ ) were used for the vibration milling experiments. The cutters were manufactured by "ASP Arno" from tungsten carbide and are coated with titanium aluminum nitride (TiAlN). This coating is suitable for high temperature and high speed machining of difficult-to-cut metallic alloys with minimal use of lubricant. Therefore this coating was chosen for our milling experiments that were carried out at high temperature without lubricating fluids. The thickness of the TiAlN coating is in the range of 2 to $4 \mu \mathrm{m}$ and the oxidation temperatures are between 480 and $900{ }^{\circ} \mathrm{C}$. The hardness is typically $2800 \mathrm{HV}$.

Each cutter has its axial resonance at different excitation frequencies due to its difference in length (14.05 and $18.4 \mathrm{kHz}$ ). These frequencies were used for tool excitation in the axial direction during vibration milling experiments, which were conducted using the following regimes: milling depth $a_{p}=1 \mathrm{~mm}$, feed rate $v_{f}=66.66 \mathrm{~mm} / \mathrm{min}$, milling speed $n_{p}=1000 \mathrm{rev} / \mathrm{min}$. Each material was cut with both mill cutters for 30 $\mathrm{mm}$ with conventional milling and then switched to $30 \mathrm{~mm}$ cutting with vibration assistance.

The surface of the workpieces was analysed qualitatively by means of a JEOL JSM-IC25S scanning electron microscope. The obtained images shown in Fig. 5 provide a visual proof that the surfaces of the workpieces machined with vibration milling are smoother with respect to the
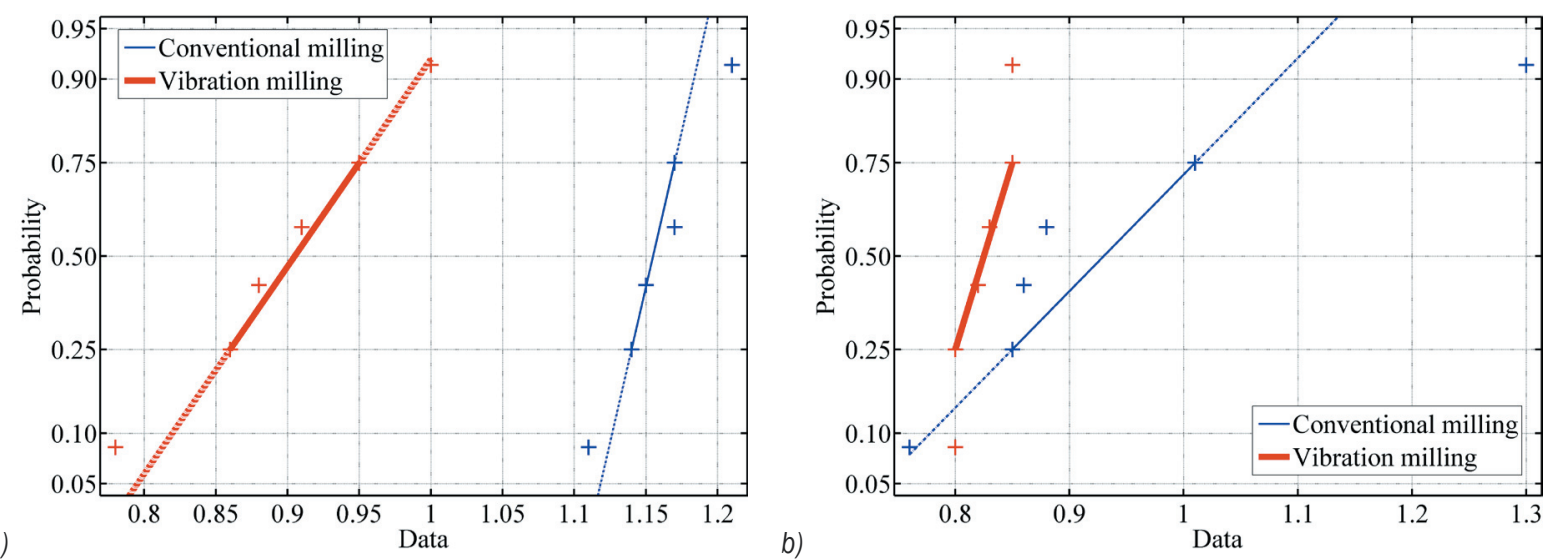

Fig. 6. Normal probability plots when $96 \mathrm{~mm}$ length cutter is used and the machined material is: a) stainless steel, b) titanium
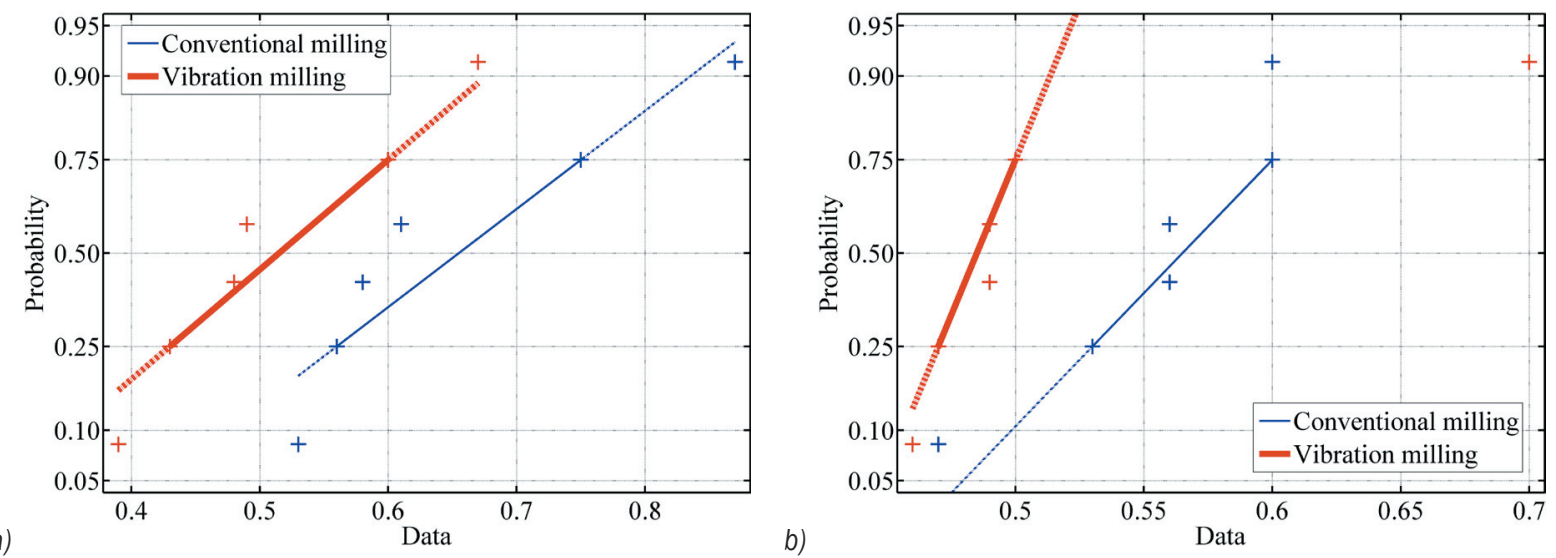

Fig. 7. Normal probability plots when $74 \mathrm{~mm}$ length cutter is used and the machined material is: a) stainless steel, b) titanium 
conventional process. The image in Fig. $5 b$ indicates no microcracks in the case of the vibration machined workpiece. A closer inspection of SEM images reveals that workpiece surface after conventional milling (Fig. 5a) is characterised by a ploughing effect due to cutting process instability. The surface in Fig. 5b, on the contrary, shows no signs of ploughing and is characterised by a regular finely meshed structure.

Quantitative results of surface quality were obtained using MITUTOYO SURFTEST SJ-201. The values of the cutoff and evaluation length were 0.8 and $2.4 \mathrm{~mm}$, respectively. Measurement data indicate that the values for surface roughness in the case of vibration milling are approximately one roughness grade number lower with respect to the conventional process (according to DIN EN ISO 1302). Surface roughness data for six machined workpieces was collected during this experimental study. Measurement results demonstrate that difficultto-cut materials machined using the vibration milling process are characterised by improved surface finish in comparison to the conventional process (Tables 1 and 2). When the tool was driven with an excitation frequency of $18.4 \mathrm{kHz}$ ( $96 \mathrm{~mm}$ cutter), the best results for the surface finish were obtained in the case of stainless steel milling (Table 2). This result is attributed to better machinability of stainless steel 309 in comparison to titanium alloy Ti 6-4.

\subsection{Statistical Analysis}

Roughness measurement data was subjected to statistical treatment in order to more thoroughly characterise the effectiveness of the vibration milling process.

As a first step, the assumption that the samples come from normal distributions was tested. A normal probability plot provides a quick idea (Figs. 6 and 7). Both data scatters approximately follow straight lines through the first and third quartiles of the samples, indicating approximately normal distributions. A shift in the mean from stainless steel and titanium machined using conventional milling to stainless steel and titanium machined with vibrations is evident.

A hypothesis test is used to quantify the test of normality. Since each sample is relatively small, a Lilliefors test is used. The test performs the default null hypothesis that the sample comes from a distribution in the normal family. The results returned by each test indicate a failure to reject the null hypothesis that the samples are normally distributed. This failure may reflect normality in the population or it may reflect a lack of strong evidence against the null hypothesis due to the small sample size.

A variance analysis was performed to estimate the relative impact of resonance frequency, applied vibrations, and material type on surface quality (Table $3)$. The $p$-value for the resonance frequency effect is 0.0159 , which is highly significant. This indicates that surface roughness is largely dependent on the dynamic characteristics of the mill cutter. In turn, this implies that in order to increase the positive influence of the vibration-assisted cutting process, the tool should be superimposed with vibrations of a frequency that corresponds to the axial resonance frequency of the cutter. As a consequence, there is amplification of axial vibration amplitudes with simultaneous intensification of the twisting motion of the cutting tip due to coupling of the axial and torsional deflections inherent to the helix-shaped mill cutter, which represents a case of parametric vibrations. The $p$-value for the vibration milling effect is 0.0471 , which is significant as well. This indicates that the surface quality also depends on the machining means. In other words, statistically, the dynamic characteristics and machining means have the greatest influence on surface quality.

\section{CONCLUSIONS}

This paper has presented an approach to reducing surface roughness of milled workpieces made from difficult-to-cut metallic alloys via excitation of the first

Table 3. Results of variance analysis

\begin{tabular}{lccccc}
\hline \multicolumn{1}{c}{ Source } & Sum Sq. & d.f. & Mean Sq. & $F$ & Prob $>F$ \\
\hline Resonance frequency & 0.32 & 1 & 0.32 & 1600 & 0.0159 \\
\hline Vibrations & 0.03645 & 1 & 0.03645 & 182.25 & 0.0471 \\
\hline Material & 0.01805 & 1 & 0.01805 & 90.25 & 0.0668 \\
\hline Resonance frequency $\times$ Vibrations & 0.005 & 1 & 0.005 & 25 & 0.1257 \\
\hline Resonance frequency $\times$ Material & 0.005 & 1 & 0.005 & 25 & 0.1257 \\
\hline Vibrations $\times$ Material & 0.00845 & 1 & 0.00845 & 42.25 & 0.0972 \\
\hline Error & 0.0002 & 1 & 0.0002 & & \\
\hline Total & 0.39315 & 7 & & & \\
\hline
\end{tabular}


axial mode of the vibration milling tool. A combined application of numerical and experimental analysis has confirmed the validity of the proposed approach. An experimentally verified finite element model of the vibration milling tool was built on the basis of the actual tool prototype. Complex tool structure in the numerical model was reduced to a single pretwisted cantilever, which was imposed with boundary conditions (clamping and excitation conditions) that accurately reproduce those of the actual vibration tool. The model was used to determine the resonance frequency of the axial vibration mode of cutters of two different lengths. Milling experiments demonstrated that excitation of the axial mode in the vibration milling tool leads to an appreciable reduction in the surface roughness of stainless steel and titanium workpieces. The application of qualitative and quantitative characterization methods revealed better surface quality in comparison to the conventional milling process: surfaces having one roughness grade lower finish were obtained. A statistical analysis of the collected roughness data allowed us to establish that the dynamic characteristics (excitation frequency) of the tool and machining method (with or without the assistance of high-frequency vibrations) have the largest effect on surface quality. The reported research results demonstrate that it is crucial to dynamically tailor the excitation frequency of the vibration cutting tool in order to generate the required vibration mode in the mill cutter and thereby achieve the most pronounced improvement in surface finish in difficultto-cut materials.

The proposed approach allows efficient machining of high-strength alloys and could significantly facilitate the treatment of hard and brittle materials such as ceramics, glass, and composite materials. The reported vibration milling experiments were successfully performed under dry machining conditions, which demonstrates that assisting cutting with high-frequency vibrations could benefit the implementation of a minimum quantity lubrication method into industrial manufacturing processes.

\section{ACKNOWLEDGMENTS}

This research work was funded by a grant from the Lithuanian Agency of Science, Innovation and Technology for the implementation of EUROSTARS project No. E!7288.

\section{REFERENCES}

[1] Kumabe, J. (1979). Vibration Cutting. Jikkyo Publishing, Tokyo.

[2] Brehl, D.E., Dow, T.A. (2008). Review of vibrationassisted machining. Precision Engineering, vol. 32, no. 3, p. 153-172, DOI:10.1016/j. precisioneng.2007.08.003.

[3] Ostasevicius, V., Gaidys, R., Rimkeviciene, J., Dauksevicius, R. (2010). An approach based on tool mode control for surface roughness reduction in high-frequency vibration cutting. Journal of Sound and Vibration, vol. 329, no. 23, p. 4866-4879, DOI:10.1016/j.jsv.2010.05.028.

[4] Twardowski, P., Wojciechowski, S., Wieczorowski, M., Mathia, T. (2011). Surface roughness analysis of hardened steel after high-speed milling. Scanning, vol. 33, no. 5, p. 386-395, DOI:10.1002/sca.20274.

[5] Chen, C-H., Wang, Y-C., Lee, B-Y. (2013). The effect of surface roughness of end-mills on optimal cutting performance for high-speed machining. Strojniški vestnik - Journal of Mechanical Engineering, vol. 59, no. 2, p. 124-134, DOI:10.5545/sv-jme.2012.677.

[6] Taylan, F., Colak, O., Kayacan, M.C. (2011). Investigation of TiN coated $\mathrm{CBN}$ and $\mathrm{CBN}$ cutting tool performance in hard milling application. Strojniški vestnik - Journal of Mechanical Engineering, vol. 57, no. 5, p. 417-424, DOI:10.5545/sv-jme.2010.059.

[7] Kubiak, K., Wilson, M., Mathia, T., Carras S. (2011). Dynamics of contact line motion during the wetting of rough surfaces and correlation with topographical surface parameters. Scanning, vol. 33, no. 2, p. 370377, DOI:10.1002/sca.20289.

[8] Imani, B., Moosavi, S. (2009). Time domain simulation of torsional-axial and lateral vibration in drilling operation. Proceedings of International Conference on Application and Design in Mechanical Engineering, Penang, Malaysia, p. 9G1-7.

[9] Zhang, J.Z, Chen, J.C. (2008). Tool condition monitoring in an end-milling operation based on the vibration signal collected through a microcontrollerbased data acquisition system. International Journal of Advance Manufacturing Technology, vol. 39, no. 1-2, p. 118-128, DOI:10.1007/s00170-007-1186-6.

[10] Seguy, S., Dessein, G., Arnaud, L. (2008). Surface roughness variation of thin wall milling, related to modal interactions. International Journal of Machine Tools \& Manufacture, vol. 48, no. 3-4, p. 261-274, DOI:10.1016/j.ijmachtools.2007.09.005.

[11] Grazeviciute, J., Skiedraite, I., Jurenas, V., Bubulis, A., Ostasevicius, V. (2008). Applications of high frequency vibrations for surface milling. Mechanika, vol. 1, p. 4649. 